\title{
Self-Assembly and Visualization of Poly(3-hexyl-thiophene) Chain Alignment Along Boron Nitride Nanotubes
}

\author{
Yadienka Martinez-Rubi ${ }^{\dagger}$,Zygmunt J. Jakubek ${ }^{\ddagger}$, Michael B. Jakubinek ${ }^{\dagger}$, Keun Su Kim ${ }^{\dagger}$, \\ Fuyong Cheng ${ }^{\dagger}$, Martin Couillard ${ }^{\#}$, Christopher Kingston ${ }^{\dagger}$, Benoit Simard $^{\dagger}$ \\ Security and Disruptive Technologies ${ }^{\dagger}$, Measurement Science and Standards ${ }^{\ddagger}$, Energy, Mining and \\ Environment ${ }^{\#}$, National Research Council Canada, Ottawa, Ontario K1A oR6, Canada
}

Estimate for the surface coverage of BNNTs by P3HT: Values of Specific Surface Area (SSA) for nanotubes and for completely stretched $\mathrm{P} 3 \mathrm{HT}$ polymer chains were used to obtain an approximate value for the BNNT surface coverage at the 0.08 rra-P3HT to BNNT weight ratio (approximately the ratio at which all available BNNT surface is covered by the polymer, after which further addition only leaves rra$\mathrm{P} 3 \mathrm{HT}$ in solution in a coil-like conformation):

$$
\text { Surface Coverage }=\frac{S S A(P 3 H T) \times 0.08}{S S A(B N N T S)}
$$

The SSA of P3HT was estimated by calculating the contour length $L_{n}=I_{O} M_{n} /\left(M_{O} B\right)$ where $I_{O}$ and $M_{O}$ are the length and molecular weight of the thiophene repeat unit. $B$ is a correction coefficient, which is about $2 .{ }^{51}$ The value of $L_{n}$ obtained for a $M_{n}$ of $34 \mathrm{kD}$ is $39 \mathrm{~nm}$. With the value of the lattice dimension of the P3HT unit cell $(1.68 \mathrm{~nm})^{\mathrm{S} 2}$ a value of SSA for P3HT of $1150 \mathrm{~m}^{2} / \mathrm{g}$ was obtained.

Depending on the assumptions made, different values of nanotube SSA can be used to estimate the surface coverage. The results are summarized in the table below and, for a reasonable range of estimates of BNNT surface area, yield qualitatively reasonable estimates for surface coverage in the range of $20 \%$ to $50 \%$ of the available BNNT surface area.

\begin{tabular}{cc}
\hline $\begin{array}{c}\text { SSA (BNNTs) } \\
\left(\mathrm{m}^{2} / \mathrm{g}\right)\end{array}$ & Surface coverage \\
\hline $500^{\mathrm{a}}$ & $20 \%$ \\
$200^{\mathrm{b}}$ & $50 \%$ \\
\hline
\end{tabular}

${ }^{\mathrm{a}}$ From reference S3 for MWNT of $4 \mathrm{~nm}$ diameter and 3 walls, which is comparable to the size of the BNNTs employed here.

${ }^{b}$ BET surface area of purified BNNTs measured experimentally 


\section{Supplementary Figures}

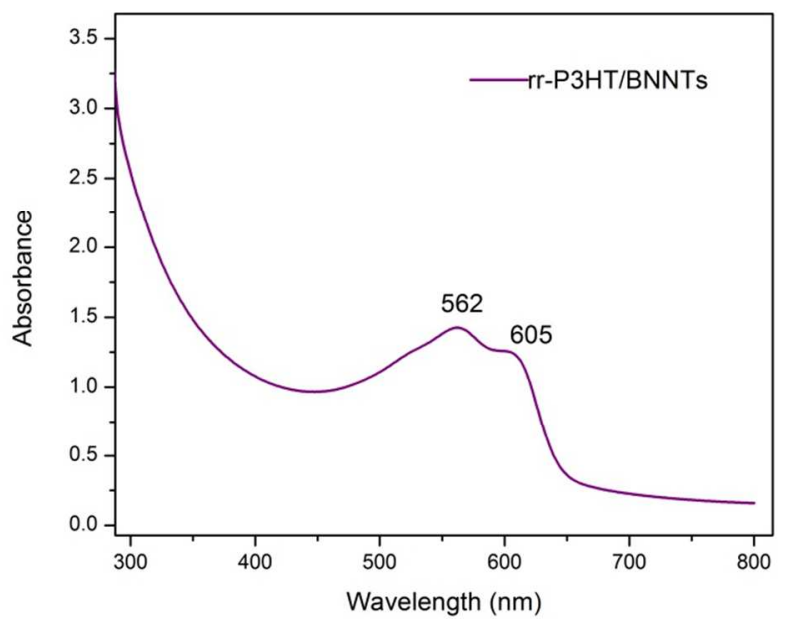

Figure S1. Absorption spectra of regioregular-P3HT/BNNT solutions. Regioregular P3HT (rr-P3HT; $\mathrm{Mw}=$ 96 kD, head-to-tail regiospecific conformation > 96\%) was obtained from Rieke Metals, Inc.

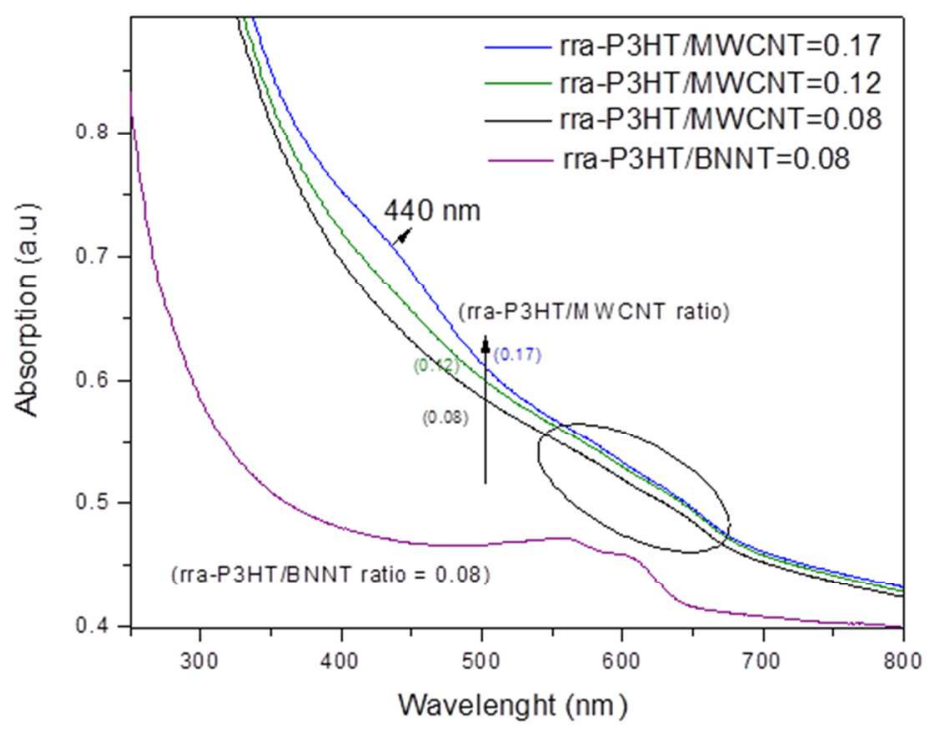

Figure S2. UV-visible absorption spectra of $0.015 \mathrm{mg} / \mathrm{mL}$ solutions of rra-P3HT/MWCNTs at different weight ratios. A weak broad band is observed $(530-680 \mathrm{~nm})$ indicating planarization and adsorption of rra-P3HT on the MWCNTs, but is less structured than for BNNT. Commercial multi-walled CNTs (NANOCYL ${ }^{\mathrm{Tm}} \mathrm{NC7000)}$ ) were used for comparisons to rra-P3HT/BNNT suspensions. 

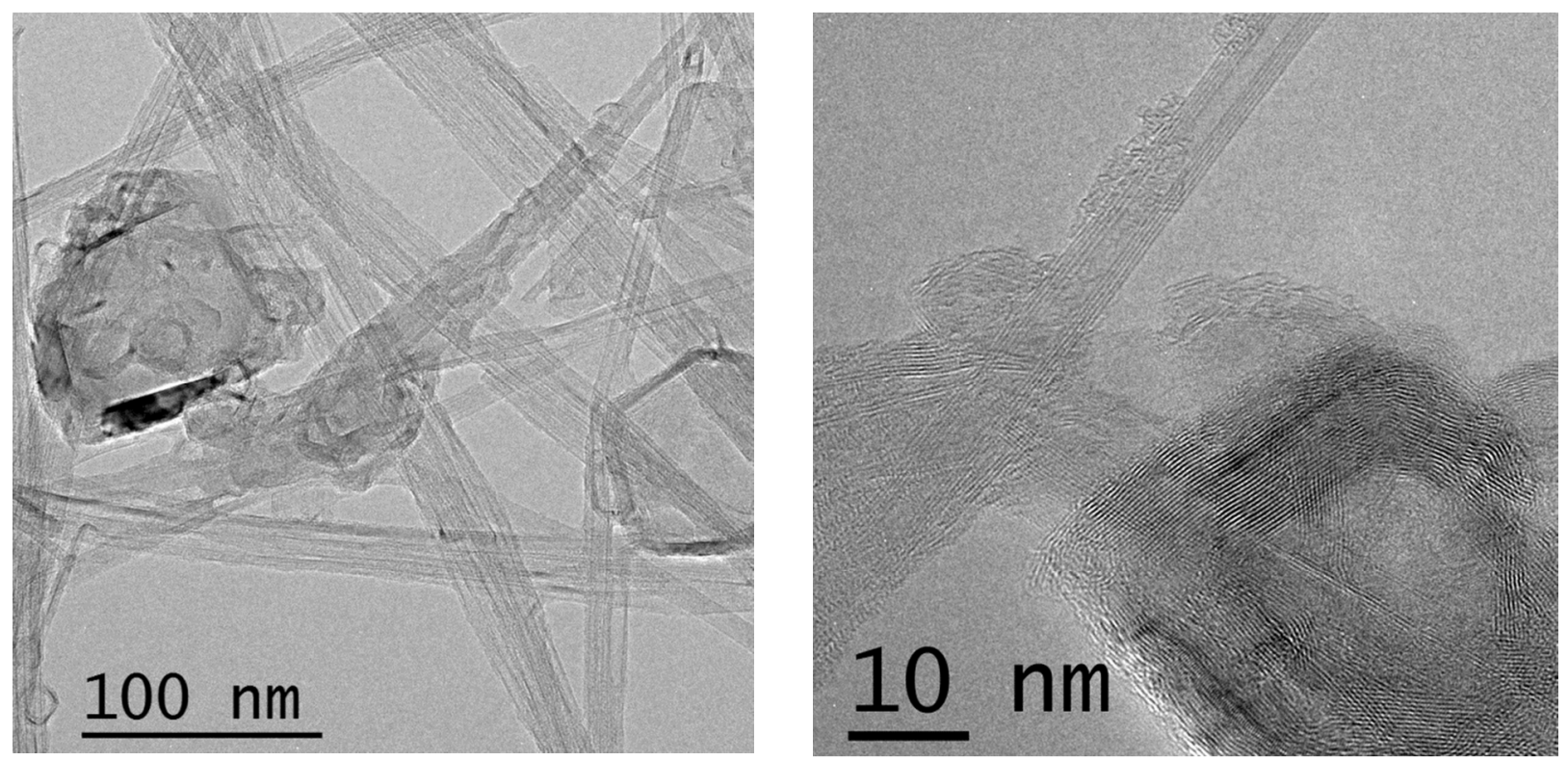

Figure S3. Drops of the solution containing purified BNNTs were deposited on a lacey-carbon support film (Ted Pella) and analyzed with a FEI Titan ${ }^{3} 80-300$ transmission electron microscope operated at $300 \mathrm{kV}$. Transmission electron microscopy images of purified BNNTs display the nanotubes and nanotube bundles, as well as some impurities. The most commonly observed impurities appear as concentric cage-like h-BN particles.

\section{Supplementary References}

(S1) Guo, Y.; Jiang, L.; Ma, X.; Hu, W.; Su, Z. Poly(3-hexylthiophene) Monolayer Nanowhiskers. Polym. Chem. 2013, 4, 4308-4311.

(S2) Liu, J.; Zou, J.; Zhai, L. Bottom-up Assembly of Poly(3-hexylthiophene) on Carbon Nanotubes: 2D Building Blocks for Nanoscale Circuits. Macromol. Rapid Commun. 2009, 30, 1387-1391.

(S3) Peigney, A.; Laurent, C.; Flahaut, E.; Bacsa, R.R.; Rousset, A. Specific Surface Area of Carbon Nanotubes and Bundles of Carbon Nanotubes Carbon 2001, 39,507-514. 